Journal of Interconnection Networks

(C) World Scientific Publishing Company

\title{
Cross-Layering for Performance Improvement in Multi-Hop Wireless Networks
}

\author{
FABRIZIO GRANELLI \\ DIT, University of Trento \\ Via Sommarive 14 Trento, I-38050, Italy \\ granelli@dit.unitn.it \\ http://dit.unitn.it/ granelli/ \\ DZMITRY KLIAZOVICH \\ DIT, University of Trento \\ Via Sommarive 14 Trento, I-38050, Italy \\ klezovic@dit.unitn.it \\ http://dit.unitn.it/ klezovic/ \\ Received (received date) \\ Revised (revised date)
}

\begin{abstract}
Cross-layering represents a perspective design principle for adapting natively wired protocols to the wireless scenario and for improving their performance. The paper explores the possibilities of cross-layer design as a candidate tool for performance optimization, focusing on problems deriving from the usage of ARQ schemes in today's wireless networks and proposes a novel and general cross-layer interaction scheme between the transport and the link layers. Achieved results in the framework of a IEEE 802.11-based multi-hop wireless network demonstrate the value of the proposed framework, and allow to provide guidelines for future work on the topic.
\end{abstract}

Keywords: Cross-layering, Multi-hop Wireless, Performance Enhancement.

\section{Introduction}

Wireless communications clearly represent a fast-growing sector in the framework of data networks ${ }^{1}$. The attractiveness of wireless networks comes from the peculiar advantages they provide and the wide range opportunities they enable. Mainly, wireless technologies provide mobile access to networks and services - omitting the requirement for a cable (and fixed) infrastructure, thus enabling fast and cost-effective network organization, deployment and maintenance.

On the contrary, the capacity offered by wireless links is relatively low as compared to wired networks. Such capacity limitations derive from the very physical nature of the wireless medium, such as limited shared bandwidth, time-varying be- 
haviour, interference, etc.

Applications of wireless solutions range from simple internet access on anytime-, anywhere-basis to systems for disaster recovery and military applications.

As a result, wireless networks created the field for new applications and services, at the same moment increasing the fruition of several existing services. Still, they suffer from several performance limitations, in some cases related to the excessive complexity deriving from the layering paradigm employed by the TCP/IP protocol stack design. Indeed, TCP/IP was originally designed for wired links which general characteristics include high bandwidth, low delay, low probability of packet loss (high reliability), static routing, and no mobility. On the contrary, in the wireless domain, performance and resource availability are limited by the availability of transmission spectrum, the employed modulation type and the limited transmission power. Indeed, loss probability experienced by packet transmission on the wireless medium are in general higher than ones on wired links: while Bit Error Rate (BER) varies from $10^{-6}$ to $10^{-8}$ for wired channels, it varies from $10^{-3}$ up to $10^{-1}$ for wireless channels ${ }^{2}$. As a consequence, high BERs of wireless channels are unacceptable for natively wired transport protocols such TCP, which additive increase multiplicative decrease (AIMD) congestion control treats all losses as congestion losses and thus underestimates the actual capacity provided by the network.

In order to counteract such range of variation in terms of BER, Forward Error Correction (FEC) can be employed at the link layer. However, FEC is not the proper solution to provide reliable transmission on wireless networks. The main drawback is the waste of transmission resources deriving from its employment in the case of absence of errors, therefore suggesting the usage of feedback information from the receiver in order to extrapolate information on the channel status. Thus, a traditional and widely implemented approach to increase reliability of wireless links is based on the usage of an Automatic Repeat reQuest (ARQ) protocol at the link layer.

ARQ provides a dynamic way to decrease error rate present on the wireless links by increasing the delivery delay. The most commonly used ARQ scheme in wireless networks is "stop \& wait": the sender is not allowed to send the next packet in the queue until the receiver positively acknowledges the successful delivery of the previous one. The advantage derives from the fact that only corrupted packets are retransmitted, introducing an overhead adapted to the conditions of the link. Scalability and low computational cost of implementation resulted in the employment of the ARQ principles in most wireless networks. In fact, it is clear that, while employing different approaches at the physical layer (in terms of modulations, data rate, occupied bandwidth), most of the existing technologies provide reliable communications by employing different ARQ schemes at the link level.

However, link layer is not the only layer which acknowledges packet delivery: TCP reliability is obtained through utilization of a positive acknowledgement scheme which specifies TCP receiver to positively acknowledge successfully received data 
from sender. TCP header reserves special fields for enabling it to carry acknowledgement information. As a result, the TCP receiver can produce a TCP acknowledgment (TCP-ACK) as standalone packet or, in case of bi-directional data exchange, encapsulate it into outgoing TCP segment.

Whenever a TCP segment is transmitted over the wireless link, the sender first receives an acknowledgement at the link layer. Then, TCP entity at the receiver generates an acknowledgement at transport layer. This acknowledgement represents ordinary payload for the link layer which should be acknowledged by the link layer of sender node. The paper introduces a cross-layering scheme in order to provide a finer ARQ procedure to enhance throughput in multi-hop wireless networks.

The structure of the paper is the following: Section 2 introduces the concept of cross-layer design applied to wireless networks, while Section 3 describes the proposed approach. Section 4 analyzes the performance of the proposed scheme through extensive simulations, while Section 5 concludes the paper and presents some outlines about future activities on the topic.

\section{Cross-Layering Design in Wireless Networks}

\subsection{Layering Principles}

Layering is a common principle in the design of communication systems, implemented in the TCP/IP protocol stack: to partition network functions into layers, and to design each layer separately. The well-known advantage of the layering paradigm lies in the creation of standard modular components for separate and independent design, thus giving full abstraction and simplifying integration.

The goal to maintain simple and scalable design as opposed to overall system optimization motivated the definition and development of a layered protocol stack for fixed cable networks. However, complete independence among protocols at different layers is not true even in the case of TCP/IP, since each layer was initially designed or later adopted with the behavior of other layers in mind. For example, the problem of finding the correspondence between network and link layer addresses motivated the design of Address Resolution Protocol (ARP). Based on ARP replies a node builds a table which contains association between network and link layer addresses.

This aspect underlines that even traditional wired networks can not be designed fully relying on the abstraction on separate layers proposed by OSI. Furthermore, wired networks possess several characteristics which simplify the design of communication protocols, such as fixed characteristics of communication channels, stable performance and no mobility. On the other hand, wireless networks are designed for mobile users and behaviour of communication channel is time-varying and highly dependant on the propagation environment. As a result, it becomes clear that potential system operation, performance, and scalability gain obtainable in wireless networks by cross-layering was reasonable enough to neglect the clarity and ease of implementation of the layered structure. 
Moreover, specific wireless functionalities such as power-aware transmission and routing, rate-adaptive communications and mobility-related handoff (handover) and roaming cannot be optimized by using a completely layered structure, since the parameters and characteristics of the different layers are to be jointly exploited.

\subsection{Cross-Layer Interactions}

Two general approaches to cross-layer interactions are "bottom-to-top" and "top-tobottom". In bottom-to-top interaction, higher layers are notified with details related to the network they operate on: a multimedia application being aware of the link capacity could adjust its sending rate by selecting an appropriate codec. Top-tobottom approach allows lower layers to access information available at the upper layers: this makes possible for network-specific layers to adjust their functionality assuming a certain behavior of application or high-level protocol running above them.

Figure 1 presents different parameters that could be exchanged in different crosslayer schemes with reference to the TCP/IP model.

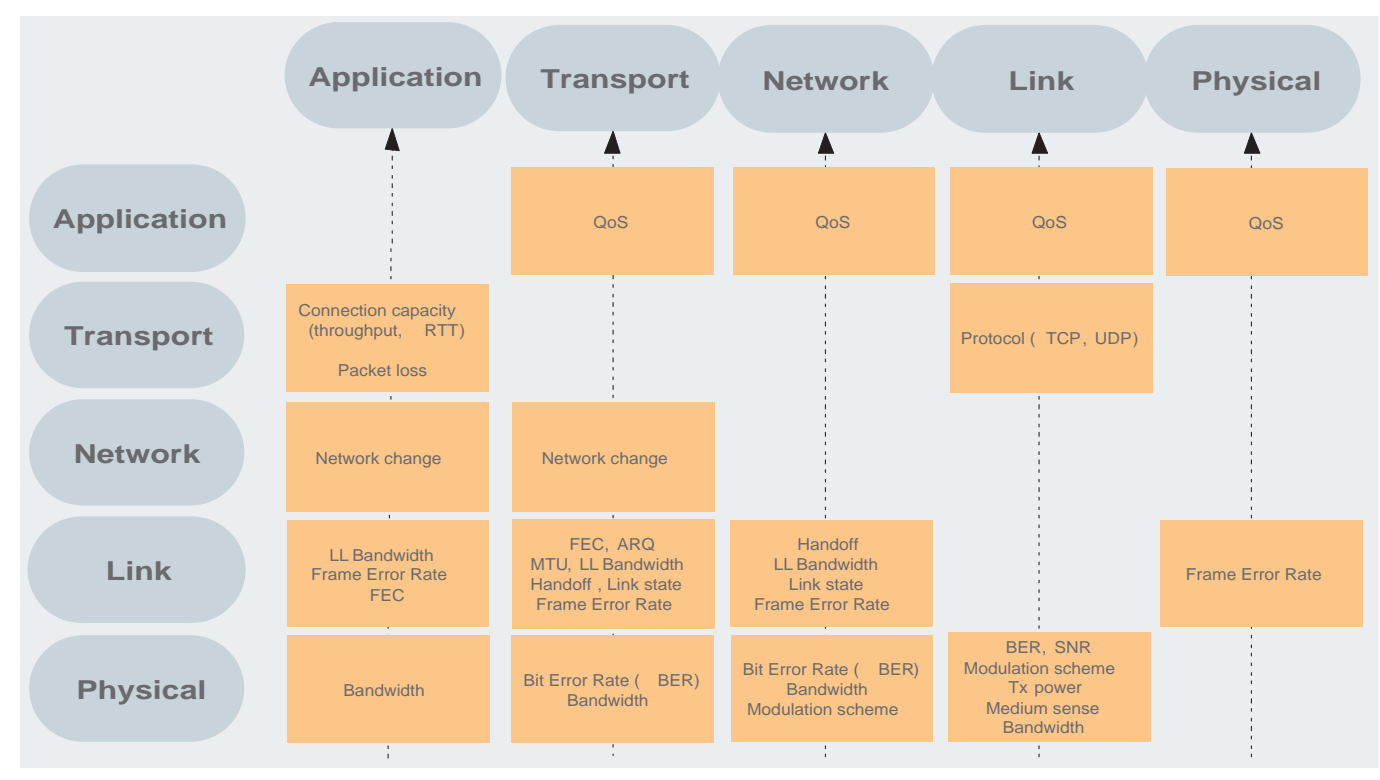

Fig. 1. Cross-layer information exchange.

Physical layer defines the means of sending data over network devices. It is characterized by transmission power, signal to noise ratio (SNR), bit error rate (BER) and available modulation schemes.

BER and SNR are important for the link layer error control mechanisms (FEC and ARQ): link layer could adjust the strength of FEC as well as the number of 
retransmissions targeting a certain BER. Transport layer performance is inversely proportional to the packet error rate (PER), which can be derived from BER or directly reported by the link layer.

Any upper layer controlling the employed modulation scheme can optimize system performance with a tradeoff between transmission range and data rate performance.

Link layer adjustment of transmission power is a key functionality for power saving mechanism, but also impacts on SNR - leading to the management of another tradeoff, between performance and battery lifetime.

Link bandwidth is one of the physical parameters which determines the maximum capacity of the network connection. If available, it could be utilized at any layer of the protocol stack: based on this parameter the network layer chooses a network route with higher capacity, transport layer adjusts its upper-bound performance, and application layer determines the transmission rate.

Link layer defines procedures for reliable operation over a communication link, by managing access to the medium, framing packets, detecting and correcting transmission errors. Link layer reports maximum frame size to upper layers for the adjustment of the packet sizes in order to avoid unnecessary fragmentation. Frame error rate could help at transport layer to adjust performance characteristics by distinguishing between link and congestion errors.

In cellular wireless networks, link layer handles handoffs between cells or between system domains (intrasystem mobility). The handoff decision, which could be forwarded to upper layers, is often based on the SNR received at the physical layer. Based on this information, network layer could initiate switching to another network and transport layer could freeze pending communications until network/link connectivity is resumed.

Channel state information is used for opportunistic scheduling which main idea is to give transmission priority to stations with better channel state.

Network layer determines how data are transferred between different network devices. It specifies the network interface a certain packet is to be transmitted onto. A guidance for network selection could be based on physical/link layer bandwidth as well as error rate information (BER or PER).

Switching between networks (intersystem mobility) in most cases takes much more time than intrasystem handoff. For that reason, transport and application layers could be notified about pending handoffs in order to avoid undesired service interruption.

Transport layer manages end-to-end message delivery over the network, providing connection-oriented reliable data flow (TCP) or simple connectionless (UDP) packet delivery.

The end-to-end connection between transport level ports constitutes a virtual circuit available for data transfer on a per-application basis. For that reason, connection capacity characteristics such as current throughput value and round trip 
time (RTT) are crucial in the determination of the application behavior since, for example, multimedia applications must adjust the quality of voice/video presentation based on the capacity information provided by transport layer.

Application layer provides an interface between the user / applications and the protocol stack of the host.

The technology designed to provide the desired operation of different user applications is Quality of Service (QoS). QoS enables allocation of network resources on a per-application basis. Application resource reservation request (required bandwidth, acceptable delay, error rate) is to be sent to all the layers of the protocol stack.

The transport layer could adjust its throughput performance selecting the proper window evolution scheme, tuning its retransmission timeouts or changing the employed ARQ scheme based on the required level of QoS. Network layer could route packets to different interfaces, differentiating between them based on link/physical layer feedback. The link layer could control the allocation of physical network resources in order to provide satisfactory QoS level.

A good example of cross-layer end-to-end QoS-enabling platform is presented in ${ }^{3}$ where authors clearly motivate the need for cross-layer design in mobile heterogeneous networks.

\section{The Proposed Scheme}

In this paper, we propose a Link Layer ARQ Exploitation TCP (LLE-TCP) approach to exploit the information of the link layer ARQ scheme for a more efficient acknowledgement of TCP packet delivery. This means that, when a TCP packet is successfully delivered at the link level, the TCP ACK for the transport layer will not be sent through the channel, but it will be automatically generated at the sender side (locally). In order to support this functionality, no changes are needed to the TCP protocol, but a new entity needs to be introduced: the ARQ agent.

The ARQ agent is a software module designed for the exploitation of the link layer ARQ scheme by TCP in wireless network environments. It operates within the protocol stack between TCP and MAC layers and it has interfaces to both TCP and MAC layers.

Figure 2a shows the logical position of the ARQ agent in the protocol stack of the wireless node. Logical attachment of ARQ agent to the link layer results in scalability.

Nowadays the link layer of wireless networks is commonly implemented inside firmware of wireless cards. An insertion of LLE-TCP functionality into the firmware releases resources of CPU simplifying integrating issues. Moreover, being attached to the link layer ARQ agent can be implemented inside wireless card driver. Both ways of implementation enable LLE-TCP operation only in desired scenarios, i.e. in wireless networks employing positive link layer ARQ. For a more detailed description of LLE-TCP on a single hop, the reader should refer to ${ }^{7}$. 


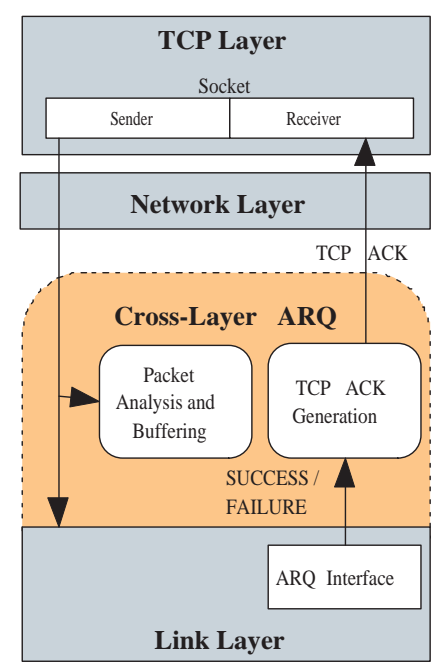

a)

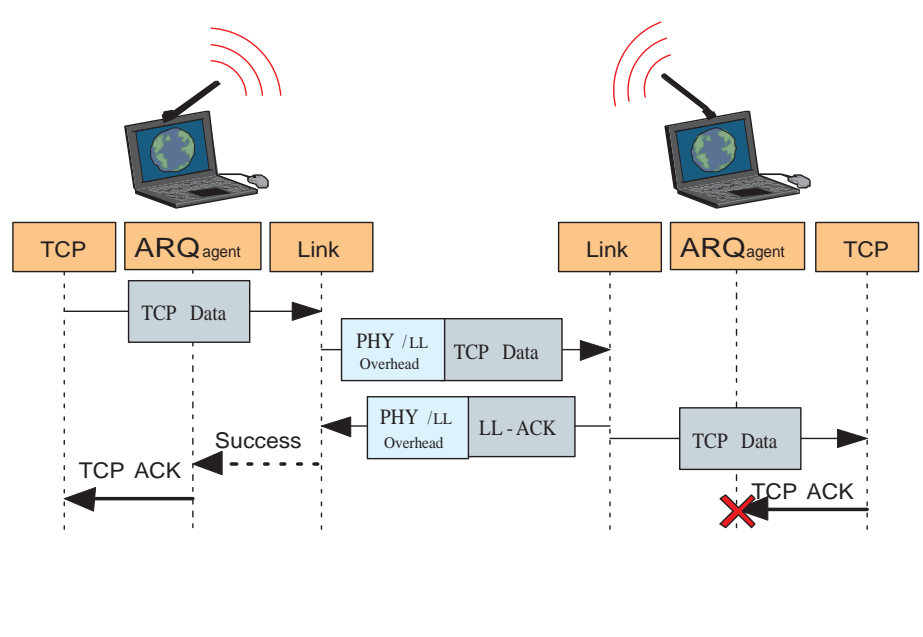

b)

Fig. 2. a) Protocol stack position and b) packet delivery diagram of the cross-layer ARQ software module.

\section{LLE-TCP in a Multi-Hop Network}

Networks consisting of wireless devices capable to operate without the requirement for fixed infrastructure form a set of ad hoc networks where data transmissions can cover several hops. Multi-hop communication being not yet standardized reflected in many research proposals ${ }^{4}$.

LLE-TCP operation is considered at the last hop of data connection. LLE-TCP agents operate at the last hop router (LHR) as well as at the receiver node (Fig. 3). Basically, while routing TCP data packets, each mobile node activates its LLE-TCP module for ACK generation if the destination is reachable within one hop. This is possible by analysis of routing table which for most of available multi-hop routing schemes contains MAC and IP addresses for at least one-hop neighborhood.

Summarizing, LHR generates end-to-end TCP ACKs back to sender node, relying on link layer acknowledgements on the last wireless hop.

Moreover, in order to support congestion control in wireless multi-hop networks, an optional LLE-TCP Congestion Control (LLE-TCP_CC) module is inserted in the protocol stack of sender node. LLE-TCP_CC moves the position of congestion control from TCP layer to itself (being located lower in the same protocol stack), adjusting the size of outgoing data through the congestion window control. LLETCP_CC locally generates for every data packet a TCP ACK for transport layer in order to get more segments from TCP. LLE-TCP_CC buffers the obtained packets maintaining reliable delivery until it receives end-to-end acknowledgement from LHR. 


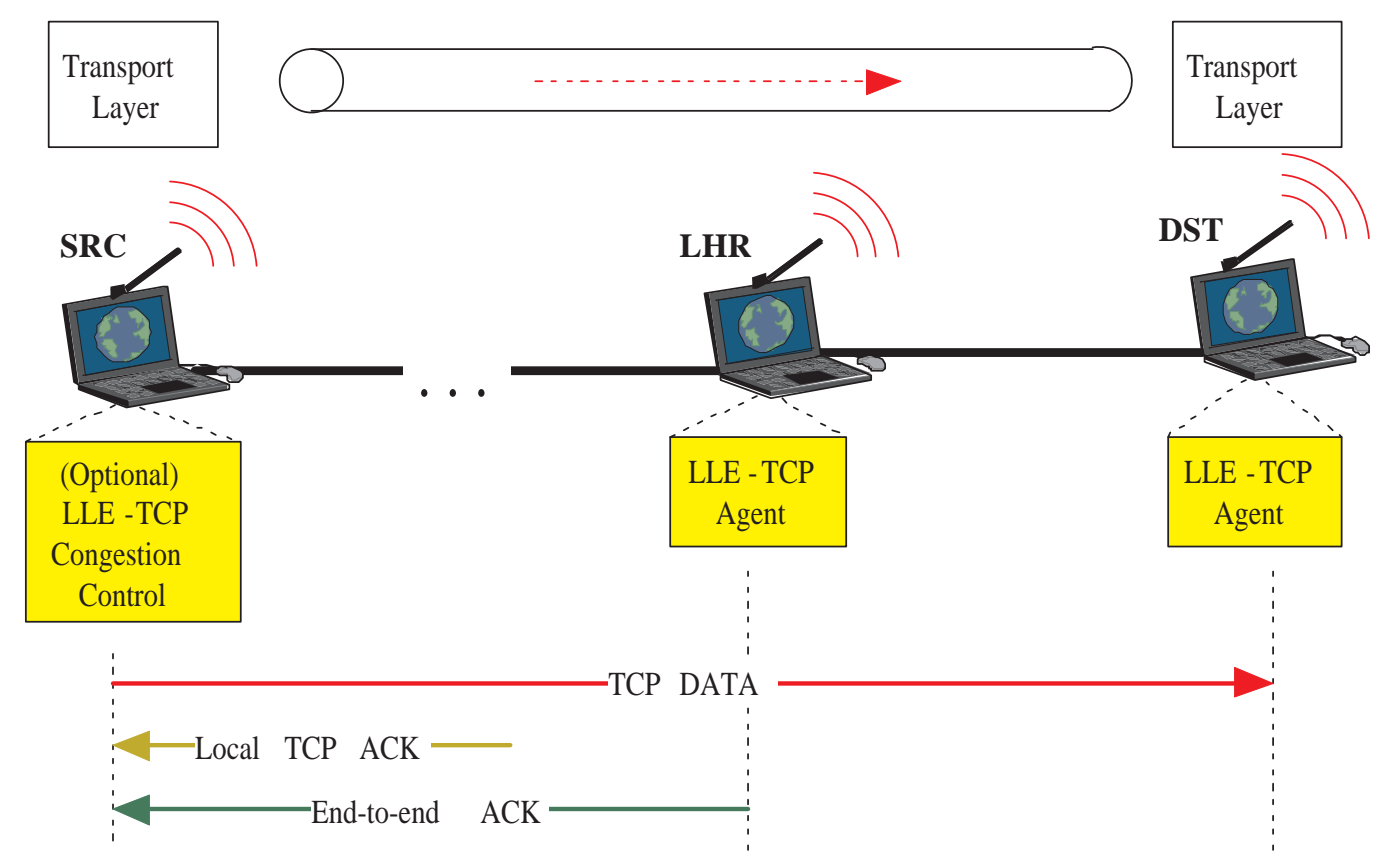

Fig. 3. LLE-TCP in multi-hop network scenario.

As a result, LLE-TCP controls the amount of data being output to the network by interfacing with TCP layer using ACK generation and rwnd techniques. Such technique is similar to C3TCP technique which also performs link layer capacity measurements (see ${ }^{5}$ for more details).

\section{Experimental Results}

Network simulator ns-2 (version 2.28 from February 2005$)^{6}$ is enhanced with software modules enabling LLE-TCP functionality. IEEE $802.11 \mathrm{~b}$ is chosen as the physical layer standard. Simulation parameters are reported in Table 1.

Table 1. Simulation Parameters.

\begin{tabular}{ll}
\hline Parameter Name & Value \\
\hline Slot & $20 \mu \mathrm{s}$ \\
SIFS & $10 \mu \mathrm{s}$ \\
DIFS & $50 \mu \mathrm{s}$ \\
PLCP preamble + header & $192 \mu \mathrm{s}$ \\
Data Rate & $11 \mathrm{Mbps}$ \\
Basic Data Rate & $1 \mathrm{Mbps}$ \\
Propagation Model & two-ray ground \\
\hline
\end{tabular}


The simulated multi-hop network consists of a variable number of static nodes arranged in string topology, where communications are allowed only between neighboring nodes due to transmission range limitations. RTS/CTS exchange is selected, in order to avoid hidden node problem.

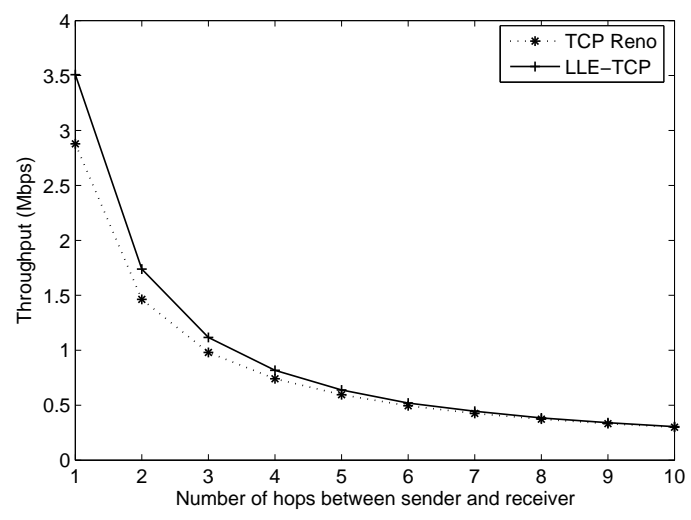

(a)

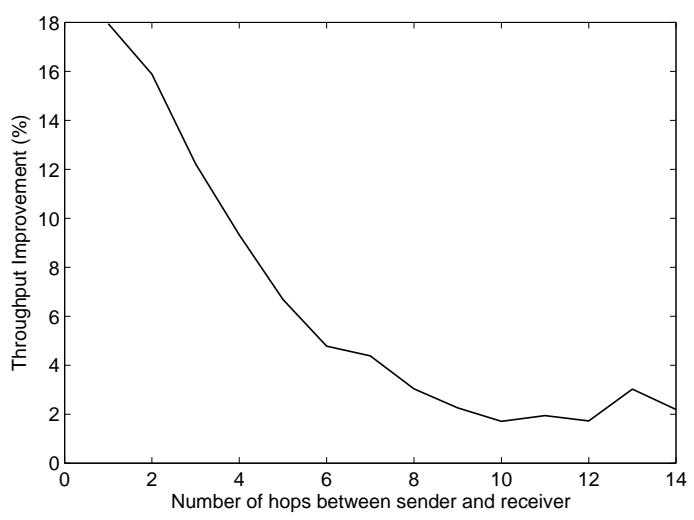

(b)

Fig. 4. a) Throughput and b) improvement of LLE-TCP in multi-hop scenario with variable number of hops.

Figure 4 presents simulation results obtained in a multi-hop scenario. TCP/IP datagram size is 1000 bytes. LLE-TCP suppresses ACKs only at the last hop of multi-hop data connection. As a result the throughput achieved be LLE-TCP is close to TCP Reno's throughput for a large number $(>7)$ of hops. However, for a smaller number of hops, it brings the throughput improvement ranging from $10 \%$ to $17 \%$.

The throughput of LLE-TCP is highly dependant on the TCP/IP datagram size since the suppressed standalone TCP ACKs are always of fixed size which corresponds to the size of packet of IP and TCP headers and no data payload. LLE-TCP throughput against datagram size is presented in Fig. 5 for four-node string topology.

Fig. 6 compares the performance of the proposed LLE-TCP agaist TCP Reno with different acknowledgement strategies employed by the receiver: one-per-packet (OneAck) and delayed acknowledgements (DelAck).

TCP Reno with DelAck receiver performs better than in case of OneAck with a low per-link packet error rate (less than 4\%). However, DelAck throughput completely degrades to zero starting from $6 \%$ of PER, while other evaluated approaches succeeds up to $8 \%$.

LLE-TCP outperforms TCP Reno modification with both OneAck and DelAck strategies in any simulation scenario. 
10 Cross-Layering for Performance Improvement in Multi-Hop Wireless Networks

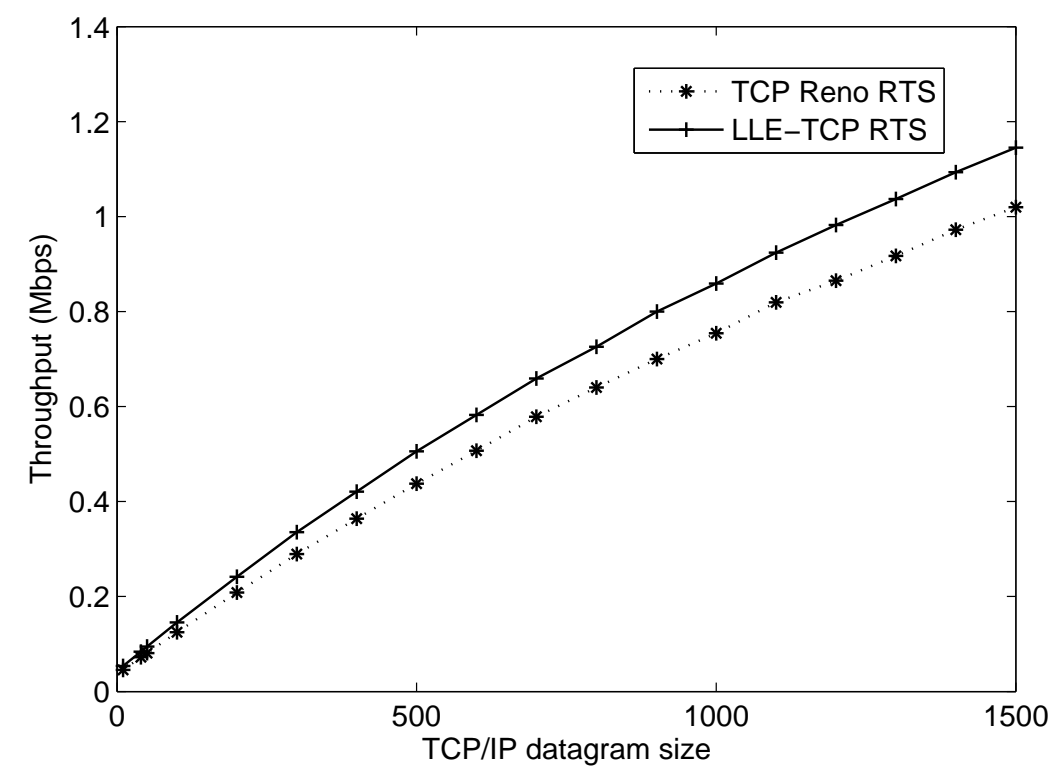

Fig. 5. LLE-TCP throughput performance against TCP/IP datagram size.



Fig. 6. Improvement level of LLE-TCP and TCP Reno with different acknowledgement strategies under packet loss conditions. 


\section{Conclusions}

The paper presented a novel cross-layering scheme to improve the coordination between the ARQ schemes employed at the transport and link layers in wireless networks. Application to wireless multi-hop networks ensures good improvements, thus enabling additional studies of the proposed architecture for gaining insight on the effective deployment of multi-hop wireless networks.

\section{References}

1. T. S. Rappaport, A. Annamalai, R.M. Buehrer, and W. H. Tranter, "Wireless communications: past events and a future perspective," IEEE Communications Magazine, 40(5) (2002), 148-161.

2. K. Pentikousis, "TCP in Wired-Cum-Wireless Environments," IEEE Communications Surveys, 3(4) (2000), 2-14.

3. Xio Gao, Gang Wu, and T. Miki, "End-to-end QoS provisioning in mobile heterogeneous networks," IEEE Wireless Communications, 11(3) (2004), 24-34.

4. I. F. Akyildiz, X. Wang and W. Wang, "Wireless Mesh Networks: A Survey," Computer Networks Journal (Elsevier), 47 (2005), 445-487.

5. D. Kliazovich and F. Granelli, "Cross-Layer Congestion Control in Ad Hoc Wireless Networks," Ad Hoc Networks Journal, (2005).

6. NS-2 simulator tool home page. http://www.isi.edu/nsnam/ns/, (2000).

7. D. Kliazovich and F. Granelli, "A Cross-layer scheme for the TCP Performance Improvement in Wireless LANs," IEEE Global Communications Conference (GLOBECOM), Dallas, U.S.A., (2004). 